\title{
Reformen des Sicherheitssektors als Voraussetzung für internationale Entwicklungszusammenarbeit
}

\author{
Wolf Poulet*
}

\begin{abstract}
After the end of the Cold War the United Nations promoted a significant shift from national security towards human security. While the USA focuses on the war against terrorism, UN, OECD and European nations emphasise security as fundamental to people's livelihoods, reducing poverty and achieving the Millennium Development Goals. When security is considered to be an essential condition for sustainable development, the role of the state and its security forces come into focus. Managing Security Sector Reforms (SSR), however, is mainly the responsibility of civil elites and civil society. Without civilian oversight over the security sector, sustainable development is not achievable. This requires new thinking and a shift of priorities of international development co-operation.
\end{abstract}

Keywords: Entwicklungszusammenarbeit, Reform des Sicherheitssektors, menschliche Sicherheit, Regierungsführung

\section{Nach Ende des Kalten Krieges ist ein neues Verständnis von Sicherheit entstanden}

$\mathrm{D}$ ie Vereinten Nationen stellten im 1994 veröffentlichten Human Development Report fest, dass das bis dahin bestehende Sicherheitskonzept zu lange eng interpretiert worden war, dass es bis dato überwiegend auf Staat/Nation ausgerichtet worden sei, nicht aber auf die Menschen selbst. »Vergessen wurden die legitimen Belange der einfachen Leute, die nach Sicherheit in ihrem täglichen Leben suchten. Für viele von ihnen bedeutet Sicherheit eher Schutz vor Krankheit, Hunger, Arbeitslosigkeit, Kriminalität, sozialen Konflikten, politischer Repression und Umweltgefährdung." Der Bericht führt weiter aus, dass »menschliche Sicherheit keine Angelegenheit der Waffen sei, sondern dass es dabei um menschliches Leben und Würde gehe«. Und er sagt voraus, dass »die Idee der menschlichen Sicherheit wahrscheinlich die Gesellschaft des 21. Jahrhunderts revolutionieren werde ${ }^{1}{ }^{1}$

Die britische Soziologin Mary Kaldor hat 1999 in ihrem Buch "Neue und alte Kriege" die Trennlinie zwischen den beiden sich entwickelnden Existenzformen auf unserer Welt beschrieben: Auf der einen Seite stehen die Angehörigen der globalen Klasse, die über Englischkenntnisse verfügen, Zugang zu Fax, E-Mail und Satellitenfernsehen haben, mit Dollar, Euro oder Kreditkarten ausgestattet sind und reisen können, wohin sie wollen. Weiterhin sind in den funktionierenden Demokratien die institutionellen Spielregeln eines intakten Sicherheitssektors so weitgehend garantiert, dass der Bürger sich ungeachtet vorhandener Kriminalität sicher fühlen darf. Vor allem aber kann er in einer offenen Marktwirtschaft für sich und die Seinen mittel- und langfristige Ent-

\footnotetext{
* Oberst a.D. Wolf Poulet, Korrespondent (Sicherheits- und Entwicklungspolitik) der Zeitschrift Entwicklungspolitik, Geschäftsführer International Governance Consulting (IGC); zuvor Referent im Deutschen Bundestag, Repräsentant einer politischen Stiftung im Ausland, Projektleiter in der GTZ (SSR), Seefeld.

1 Entwicklungsprogramm der Vereinten Nationen, Bericht zur menschlichen Entwicklung 1994, New York, 1994, S. 22.
}

scheidungen und Investitionen mit garantierten Eigentumsrechten tätigen.

Demgegenüber befinden sich auf der anderen Seite jene, die von globalen Prozessen ausgeschlossen sind; die von dem leben, was sie verkaufen oder eintauschen können oder an humanitärer Unterstützung erhalten; deren Bewegungsfähigkeit durch Straßensperren, Visa und hohe Reisekosten begrenzt ist und die von Belagerungen, erzwungenen Hungersnöten, Landminen und ähnlichem bedroht sind. ${ }^{2}$ Auch nach vorsichtigen Schätzungen muss die Mehrzahl der Weltbevölkerung unter solchen Prämissen als »Marginalisierte leben. Die Eliten in solchen Länder bringen aus leicht nachvollziehbaren Gründen wenig Interesse an der Verbesserung der sozialen Aspekte auf. Die Justiz steht in vielen Entwicklungsländern eindeutig auf der Seite der Mächtigen. Polizei und Militär sind unterbeschäftigt, schlecht ausgebildet wie auch unterbezahlt und betreiben oft des schlichten Überlebens willen - den materiellen Bestand ihrer eigenen Familien durch eine Strategie, die man hierzulande als Korruption bezeichnet.

Es stellt sich nun die Frage: Warum hat sich die Weltgemeinschaft trotz der offensichtlich durchaus vorhandenen Einsicht bisher nicht zu einer wirksamen Umgestaltung der internationalen Entwicklungszusammenarbeit verstehen können, um die persönliche Sicherheit der Menschen in Entwicklungsländern verbessern?

\subsection{Der Terrorismus bindet als »neuer Feind« das internationale Engagement der USA}

Die nach Ende des Kalten Kriegs anstehende Umorientierung von äußerer (militärischer) Sicherheit auf die innere (menschliche) Sicherheit wurde maßgeblich gehemmt durch den stetig schärfer werdenden Konflikt zwischen der einzigen

2 Mary Kaldor, Neue und alte Kriege. Organisierte Gewalt im Zeitalter der Globalisierung, Frankfurt am Main 2000. 
Weltmacht USA und dem islamistischen Extremismus, offen erkennbar nach dem ersten Anschlag auf das World Trade Center in New York im Februar 1993. Während in Europa zunehmend von einem »erweiterten Sicherheitsbegriff « gesprochen wurde, nach dem Sicherheit als ein Komplex von mindestens fünf Faktoren aufzufassen wäre (»ökonomische, soziale, ökologische, innere und äußere Sicherheit«), bereiteten sich die USA mit einem militärisch ausgerichteten Konzept auf die Abwehr terroristischer Angriffe vor. Aus dieser Logik heraus erklärten sie nach dem Anschlag am 11. September 2001 dem »Terrorismus« den Krieg.

Wer die Entwicklung in den USA verfolgt hat, kann nachvollziehen, dass sich die Weltmacht auf vielfältige Art und Weise und unter Einsatz enormer Ressourcen auf die Abwehr möglicher Anschläge konzentriert. Der Hafenkapitän von Los Angeles berichtete kürzlich im TV-Sender CNN, dass von den etwa 22.000 täglich über die Seehäfen in die USA eingehenden Fracht-Containern nur ein bis zwei Prozent kontrolliert werden könnten. Seine Folgerung daraus: »Wenn jemand etwas Übles in das Land einführen will, hat er es längst getan. «So ist es nachvollziehbar, dass auf einer Anfang Mai 2005 in McLean bei Washington D.C. (erstmalig offen) durchgeführten internationalen Konferenz der US-Nachrichtendienste die Thematik der »human security außerhalb der USA « kein Thema war. ${ }^{3}$

Die terroristische Bedrohung gilt auch für Europa und alle übrigen Erdteile. Allerdings wird außerhalb der USA die Gefahrenlage differenzierter eingeschätzt, und man hat auch abweichende Vorstellungen darüber, wie mehr Sicherheit erreicht werden könnte.

\subsection{Die Reform des Sicherheitssektors als neues Thema für das 21. Jahrhundert}

Bereits in den 1990er Jahren hatten UNDP (United Nations Development Programme) und die OECD (Organisation for Economic Co-operation and Development) den Zusammenhang von Sicherheit und Entwicklung gründlich untersucht. Auch die britische Regierung hatte diese Thematik frühzeitig verfolgt, der Terminus »security sector reform «soll 1998 von der damaligen Entwicklungsministerin Claire Short kreiert worden sein. ${ }^{4}$ Vor allem aber der Entwicklungsausschuss (Development Assistance Committee, DAC) der OECD, mit Sitz in Paris, hatte im Jahr 2001 jahrelang betriebene Untersuchungen in einer Richtlinie zur Internationalen Entwicklung, »Helping Prevent Violent Conflict", zusammengefasst und dabei grundlegende Erkenntnisse zum Thema »Reform des Sicherheitssektors (SSR)« vorgelegt. Die primäre Zielsetzung war dabei aber noch auf die (zivile) Konfliktprävention gerichtet, die als »integraler Bestandteil der Verfolgung von Armutsreduzierung « betrachtet wurde. ${ }^{5}$

3 Siehe unter https://analysis.mitre.org/.

4 Clare Short, »Security, Development and Conflict Prevention«. Speech at the Royal College of Defence Studies, London, 13 May 1998.

5 Organisation for Economic Co-operation and Development (OECD), Helping Prevent Violent Conflict, Orientations for External Partners, Paris 2001, http://www.gm-unccd.org/FIELD/OthOrg/OECD/Conflicts.pdf.
UNDP veröffentlichte Ende 2002 ein Papier zur Justiz- und Sicherheitssektorreform (JSSR), in dem der Zusammenhang von Entwicklung auf der einen und JSSR auf der anderen Seite herausgestellt und darüber hinaus in den Kontext von Konfliktprävention und Friedensentwicklung gestellt wurden. ${ }^{6}$

Ein neues Grundlagenpapier zu SSR, »Security System Reform and Governance", wurde im Mai 2004 von DAC/OECD veröffentlicht. Dort wird nicht mehr Krisenprävention in den Mittelpunkt gestellt, sondern die menschliche Sicherheit der Bevölkerung, verbunden mit der Verpflichtung $\mathrm{zu}$ "guter Regierungsführung « und der (nur) damit möglichen Erreichung der Millennium Development Goals (MDG) der UN im Zentrum der Erfordernisse gesehen. Als erste internationale Stiftung hat sich DCAF (Geneva Center for the Democratic Control of Armed Forces) seit 2000 die Aufgabe gestellt, die Reform und die demokratische Regierungskontrolle des Sicherheitssektors in den Staaten, die hier Bedarf haben, zu unterstützen. Die Entwicklung der internationalen Politik gibt seit dem September 2001 wenig Hoffnung auf die Erfolgschancen einer (möglichst zivilen) Krisenprävention, deren Instrumente bisher unscharf geblieben sind und deren Prinzipien von den stärksten Mächten im globalen Maßstab bisher nicht akzeptiert werden. Demgegenüber hat die Einsicht in die Bedeutung von »Human Security « und dorthin führender Sicherheitssektorreform zugenommen. Im Verlauf einer Veranstaltung des Auswärtigen Amtes am 11. Mai 2005, aus Anlass des Jahrestages des Aktionsplans der Bundesregierung »Zivile Krisenprävention, Konfliktlösung und Friedenskonsolidierung «, stellte Christoph Heusgen, Direktor der Strategie- und Frühwarneinheit im Kabinett von Javier Solana, fest, dass auch aus präventiver Sichtweise die Reform des Sicherheitssektors das zentrale neue Thema sei.

Warum hat dieses »operative Handlungsfeld menschlicher Sicherheit« aber erst so spät Eingang gefunden in die Kernbetrachtung der europäischen Außen-, Sicherheits- und Entwicklungspolitik?

\subsection{Die Definition von SSR ist noch weitgehend unbekannt}

Obgleich Think-Tanks verschiedene Definitionen von SSR anbieten, scheint sich die vom Entwicklungsausschuss der OECD vorgelegte weitgefasste Definition von SSR (wobei der Entwicklungsausschuss SSR mit Sicherheitssystemreform bezeichnet) zunehmend durchzusetzen. Sie wurde auch von den zuständigen Stellen im Bereich der EU übernommen, ebenso fast wortgleich von den Vereinten Nationen (UNDP) und dem britischen Department for International Development, DFID. Mit der Annahme des erwähnten Grundlagenpapiers der OECD vom Mai 2004, die auch durch die Vertreter der Bundesregierung erfolgte, hat diese Definition auch für den deutschen Bereich Gültigkeit. Die Kenntnis der Definition ist von Bedeutung, weil der Terminus SSR noch vielfach als bloße

6 United Nations Development Program, Justice and Security Sector Reform. BCPR's Programmatic Approach, New York, November 1992, http://www.undp.org/bcpr/jssr/. 
Reform von Polizei und Militär eingestuft wird - befreite man diese vom Geist der Vergangenheit, würden sich schon die gewünschten Resultate einstellen.

Nach der nun als gültig angenommenen Definition des DAC gilt: »Sicherheitssystemreform ist ein Begriff für die Beschreibung der Transformation des Sicherheitssystems - mit allen seinen Akteuren, ihren Rollen, Verantwortlichkeiten und Aktionen - in Richtung auf ein einheitliches Führen und Handeln des Sektors, das demokratischen Normen und Prinzipien der guten Regierungsführung besser entspricht und damit zu gut funktionierenden Rahmenbedingungen des Sicherheitsbereichs beiträgt. «7

Das Sicherheitssystem umfasst nach der Definition des Entwicklungsausschusses:

Zentrale Sicherheitsakteure: Streitkräfte, Polizei, Gendarmerie, paramilitärische Kräfte, Präsidentengarden, Nachrichtenund Sicherheitsdienste (militärisch und zivil), Küstenschutz, Grenzschutz, Zollbehörden, Reserve- und lokale Sicherheitseinheiten (Zivilschutzkräfte, Nationalgarden, Milizen).

Management- und Aufsichtsorgane für Sicherheit: die Exekutive, nationale Sicherheitsberatungsorgane, die Legislative und ihre entsprechenden Ausschüsse, Ministerien für Verteidigung, Innere Angelegenheiten, Auswärtige Beziehungen; überkommene und traditionelle Autoritäten, Organe für finanzielles Management, Haushaltsbüros, Finanzkontroll- und -planungsbehörden, und Organisationen der Zivilgesellschaft (zivile Eingabestellen und öffentliche Beschwerdekommissionen).

Institutionen der Justiz und Rechtsdurchsetzung: Gerichte, Justizministerien, Gefängnisse, Staatsanwaltschaften, Menschenrechtskommission und Ombudsmänner, überkommene und traditionelle Justizsysteme.

Nicht-verregelte Sicherheitskräfte, mit denen Entwicklungsorganisationen selten zu tun haben: Befreiungsarmeen, Guerillaarmeen, private Personenschützer, private Sicherheitsfirmen, Milizen politischer Parteien.

Anschließend wird aus den vorhergegangenen DAC Guidelines »Helping Prevent Violent Conflicts « zitiert: »We recognise the need to help the partner countries build legitimate and accountable systems of security to prevent violent conflict. This is an integral aspect of good governance and public sector management. Security reform includes promoting transparency, the rule of law, accountability and informed debate, and reinforcing legislative capacity for adequate oversight of security systems. Security reform involves a range of actors from the military and the police, to judicial and penal systems, ministries of foreign affairs, trade, commerce and civil society organisations...«8

Diese weitgefasste Definition ist bisher in Fachkreisen und

7 »Security system (i.e. sector) reform is another term used to describe the transformation of the security system - which includes all the actors, their roles, responsibilities and actions - working together to manage and operate the system in a manner that is more consistent with democratic norms and sound principles of good governance, and thus contributes to a wellfunctioning security framework. " OECD DAC, Security System Reform and Governance, Paris 2004, S. 19, http://www.oecd.org/dataoecd/8/39/31785288.pdf.

8 Anm. 5, S. 16. politischen Gremien Deutschlands so gut wie nicht diskutiert worden. Auf internationaler Ebene existieren umfangreiche Vorstellungen, Konzepte, Untersuchungen, Umfragen und Grundlagenpapiere zu SSR. Einzelne Regierungen der EU, allen voran das Vereinigte Königreich, aber auch die Niederlande, haben bereits konkrete Vorstellungen zur Umsetzung entwickelt. In der aktuellen internationalen Diskussion ist man sich einig, dass die Reform des Sicherheitssektors untrennbar verbunden ist mit dem Streben nach Demokratie und »Guter Regierungsführung «. UN-Generalsekretär Kofi Annan hatte bereits 1999 in einem Brief an die Weltbank darauf verwiesen, »another very important aspect is the reform of public services - including the security sector, which should be subject to the same standards of efficiency, equity and accountability as any other service.$^{9} 9$

Der wichtigste Faktor zum Verständnis von SSR liegt darin, dass dieser Reformprozess nur zum Teil Aufgabe der Sicherheitsorgane ist, primär aber eine Führungsaufgabe der zivilen Eliten in Staat und Gesellschaft.

Diese müssten SSR als ihre Verpflichtung ansehen und sowohl für die Umgestaltung als auch für die bürgergerechte Funktionserfüllung einen Hauptanteil der Verantwortung übernehmen. Ein von außen aufoktroyierter Reformprozess im Sicherheitssektor würde in jedem Fall zum Scheitern verurteilt sein. Damit ergeben sich auch für die Geberländer neue Ansätze, etwa in der - nunmehr unausweichlichen Verlagerung des eigenen Schwerpunkts in den komplexen Bereich von Governance. Governance-Beratung ist aber ohne Einbeziehung von SSR in das Reformprogramm praktisch wertlos, wenn nicht sogar ein Verstoß gegen das Prinzip von Do no Harm. ${ }^{10}$ Die zentrale Frage ist somit: Wie kann man Ziele und Bedingungen einer SSR in der internationalen Entwicklungszusammenarbeit verankern, mit denen sowohl die Richtlinien der Geberländer als auch die politischen Visionen des jeweiligen Entwicklungslandes miteinander verknüpft werden können?

\section{Exkurs in die gesellschaftliche Praxis von nicht-funktionierenden Sicherheitssektoren}

\subsection{Beispiele}

Zeitungsnotiz aus einem südamerikanischen Land, das sich im Wahlkampf befindet und dessen autoritär regierender Staatschef mit allen Mitteln nach Wiederwahl strebt: In einer Gaststätte will ein betrunkener Ex-Polizist einen Saufkumpan davon abhalten, schon nach Hause zu gehen. Er feuert mit seiner Pistole mehrere Schüsse in Luft ab, ein Querschläger

\footnotetext{
9 Ein umfassender Überblick dazu wird geboten in Heiner Hänggi und Alan Bryden (Hrsg.), Reform and Reconstruction of the Security Sector, Münster 2004; siehe auch den Beitrag von Heiner Hänggi in diesem Heft.

10 Das Prinzip, Maßnahmen vorab daraufhin zu prüfen, ob sie keinen Schaden anrichten, ist in der Krisenprävention weit verbreitet: vgl. Mary B. Anderson, Do no Harm: How Aid Can Support Peace - or War, Boulder 1999.
} 
verletzt einen kleinen Jungen lebensgefährlich. Der Täter geht anschließend unbehelligt nach Hause, es werden keine Zeugen befragt, die Staatsanwaltschaft führt keine Ermittlungen durch. Causa finita.

Fortsetzung: Dieser Vorfall wird von einer Gruppe von Politikberatern aufgenommen, die einen Gegenkandidaten wahlstrategisch unterstützen. Man weist darauf hin, dass der amtierende Präsident seine Unersetzlichkeit auch damit unterstreicht, dass er sich als Garant der Sicherheit im Lande darstellt und hier nun ein Beispiel von staatlich tolerierter Un-Sicherheit für den einfachen Bürger vorliegt. Der unterstützte Kandidat lehnt eine Befassung mit dieser Sache $\mathrm{ab}$, offenkundig weniger aus Angst vor Repressionen der Staatsmacht als vielmehr aus Unverständnis des Zusammenhangs.

In einem karibischen Land werden drei jugendliche Straftäter nach einem Feuergefecht mit der Polizei gefesselt auf einen Pritschenwagen verladen. Auf dem nächsten Polizeirevier angekommen, sind die Jugendlichen tot, Kopfschuss. Die Sache wird publik, weil zufällig ein Passant mit der Videokamera die jungen Männer filmte, als diese noch lebend auf dem LKW lagen. - Die Polizisten werden vor Gericht gestellt und erhalten niedrige Freiheitsstrafen. Es konnte nicht ermittelt werden, wann sie tatsächlich wieder auf freien Fuß gesetzt wurden.

In einem mittelamerikanischen Land zeigt ein junger Jurist, der Polizeichef des wichtigsten Bezirks der Hauptstadt (MegaCity), ein Foto mit seinen fünf direkt unterstellten Polizeiführen. Er berichtet, dass vier der Polizisten über eigene Banden und kriminelle Bereiche verfügten, er aber bisher nichts dagegen habe tun können, da diese über Rückendeckung »von oben« verfügten. Er macht deutlich, dass er weiter Beweise sammeln werde.

In einem osteuropäischen Land wird ein Beauftragter der EU, ein deutscher Offizier, am letzten Tag der Kontrollfunktion in einer Provinzhauptstadt von seinen Begleitern zu einem Abschiedsessen eingeladen. Man geht in ein neu eröffnetes Restaurant, das von einem jungen jüdischen Ehepaar betrieben wird. Während des Essens füllt sich der Raum mit zahlreichen muskulösen jungen Männern. Die Begleiter entstammen dem Militärgeheimdienst und vermitteln ihrem Gast, dass er sich keine Sorgen machen solle. Kurz nachdem er mit seiner Begleitung das Restaurant verlassen hat, kann man von außen der Zerstörung des Restaurants zusehen und -hören. Die Begleiter lehnen eine Intervention ab.

Diese Beispiele stellen noch nicht einmal die Spitze des Eisbergs dar. An jedem Tag geschehen auf allen Kontinenten und in allen Ländern, die nicht über ein staatlich und demokratisch kontrolliertes Gewaltmonopol verfügen, aus diesem Grund unzählige Verstöße vergleichbarer oder noch schwerwiegenderer Art.

\subsection{Welche Merkmale einer schwachen Staats- struktur mit unzureichend funktionierendem Sicherheitssektor können aus den aufgeführ- ten Vorfällen abgeleitet werden?}

Erstens: Durch die gesetzwidrige Zusammenarbeit oder gegenseitige Duldung von Justiz, Polizei, Strafverfolgungsbehörden und politischen Entscheidungsträgern führt ein schlecht funktionierender Sicherheitssektor zwangsläufig zur Verhinderung einer positiven Weiterentwicklung in Richtung auf mehr »menschliche Sicherheit «.

Hier wird Komplexität und Sensitivität des SSR-Prozesses deutlich, ebenso wie die unbedingte Erfordernis einer Zustimmung (»Ownership«) aller genannten Gruppen. Nach den Vorstellungen der OECD bieten sich »Security Sector Reviews« an, um die Grundeinstellung der Akteure im Land einschätzen und somit Entscheidungen sinnvoll vorbereiten zu können.

Möglicherweise ist das Verhindern der Entstehung oder die Unterdrückung einer Mittelschicht das entscheidende negative Merkmal. Eine solche Mittelschicht - quasi als Hefe des Reformprozesses - entstehen und wachsen zu lassen, könnte ein bedeutsamer neuer Beitrag der Entwicklungszusammenarbeit sein. Es ist zu bezweifeln, ob Entwicklungszusammenarbeit überhaupt nachhaltig sein kann, wenn zum Beispiel eine Landreform mit Unterstützung von außen eingeleitet wird, die Geber das Land verlassen und danach auf Grund allgemeiner Rechtlosigkeit ungestraft das Reformergebnis unterhöhlt oder annulliert werden kann.

Zweitens: Bei der Oberschicht in zahlreichen Ländern hält sich die Empathie mit dem Los der verarmten Mehrheit in Grenzen. Diese Beobachtung gilt nicht pauschal, ist aber häufig anzutreffen. Die Möglichkeiten der Einflussnahme von außen sind begrenzt. Eine Verhaltensänderung erscheint nur dann annehmbar, wenn die konkreten Lebensumstände der Betroffenen tangiert würden. Hier könnte im Rahmen der Entwicklungszusammenarbeit wirksam unterstützt werden, etwa durch diplomatische und vertrauliche Einzelberatungen, geleistet von Politikern und Beratern mit sicherheitsbezogenem Hintergrund.

Die Reformbereitschaft nationaler Eliten wird auch durch gesellschaftliche Veränderungen verstärkt, die sich - wenn auch zunächst kaum messbar - durch bereits eingeleitete Einzelmaßnahmen im SSR-Bereich vollziehen. Gelingt es z.B. das Wahlrecht für Polizei/Militär als Einzelmaßnahme einzuführen, wird die bisherige »Prätorianerrolle« dieser Institutionen abgeschwächt - Untersuchungen über den Wirkungsgrad solcher Einzelreformschritte stehen jedoch noch aus. Man kann aber davon ausgehen, dass die Globalisierung die Notwendigkeit oder gar den Zwang zur Reform befördert.

Drittens: Es herrscht Freiheit von Strafverfolgung, Impunity, Impunidad. Angehörige oder ehemalige(!) Angehörige der uniformierten Ordnungskräfte sind einer allgemeinen und konsequenten Strafverfolgung entzogen. Dies hat zur Folge, dass die große Mehrheit der Bürger gegenüber diesem Personenkreis rechtlos ist, im öffentlichen wie im privaten Raum. Die weitgehende Freiheit von Strafverfolgung fördert 
und verstärkt die Straffälligkeit im Einzelnen wie allgemein. Insgesamt entsteht ein rechtloses Staatswesen, in dem nur korrupte »Ordnungshüter « und diejenigen, die entsprechende Mittel aufbringen, mit persönlicher Sicherheit rechnen können. Die übrigen Bürger sind in allen Lebensbereichen sei es Existenzgründung, Fürsorge für Alter oder Familie, Zugang zu Bildungseinrichtungen, freies wirtschaftliches Handeln u.v.m. erheblich benachteiligt und verbleiben daher in der Mehrzahl im informellen Sektor, in abhängigen und nicht exponierten Positionen. Für die Entwicklung der jeweiligen Gesellschaft bringt dies das Absterben von Eigeninitiative (außer im kriminellen Milieu), kreativem Denken und Handeln, von Verantwortungsgefühl und Zivilcourage mit sich.

Hier gilt es anzusetzen. Die Entwicklungen der sich globalisierenden Welt sind günstig für Diskussion und spätere Akzeptanz von Reformprozessen. Im globalen Wettbewerb werden diejenigen Staaten, die sich den neuen Informationsund Produktionsbedingungen schneller und umfassender als ihre natürlichen Konkurrenten öffnen, als Gewinner hervorgehen.

\section{Governance als Voraussetzung einer nachhal- tigen Entwicklungszusammenarbeit}

Die internationale Entwicklungszusammenarbeit ist in unüberschaubare Einzelaspekte aufgefächert. Ihre hochgesteckten Ziele begründen sich darauf, dass der weitgehend rational organisierte und wohlhabende Teil der Staatenwelt ein nachvollziehbares Interesse hat, "zur Schaffung menschenwürdiger Lebensverhältnisse für alle Menschen - Mann oder Frau in den Entwicklungsländern beizutragen «. ${ }^{11}$

Man kann der so genannten OECD-Welt drei grundsätzliche Beweggründe für ihren Einsatz von Personal und Ressourcen in der internationalen Zusammenarbeit unterstellen. Erstens der altruistische Aspekt: Wer mehr hat, kann und sollte davon abgeben. Im Hintergrund stehen christliche oder andere religiöse/humanitäre Weltanschauungen. Zweitens der egoistische Aspekt: Je mehr Länder in Richtung auf ein Staatsversagen zutreiben, desto mehr verlieren die Staaten der »Ersten Welt« ihre Kooperationspartner und Märkte. Deshalb »lohnt « sich die Hilfe. Und drittens der Sicherheitsaspekt: Je weniger Länder in die Unregierbarkeit abstürzen, desto geringer sind die Chancen zur Einrichtung von Einsatzund Sammelräumen für internationale Kriminalität und Terrorismus.

Die These, dass die Reform des Sicherheitssektors als grundlegende Voraussetzung jeder weiteren Entwicklungszusammenarbeit angesehen werden sollte, setzt sich zunehmend durch.

Bilaterale und multilaterale Entwicklungszusammenarbeit, die nicht zuerst eine Kooperation im Bereich von »Govern-

11 Ansprache von Staatssekretär Erich Stather, BMZ, bei der Tagung »Eindämmung der grenzüberschreitenden Kriminalität - Herausforderungen für die Internationale Entwicklungszusammenarbeit«, Bonn, 16.12.2002. ance prüft, wird, so die Vorhersage, in wenigen Jahren von den Gebern nicht mehr geleistet werden können und wollen. Ausgenommen hiervon bleibt die internationale Nothilfe, wie kürzlich nach dem Tsunami.

Zur Überprüfung dieser These wird eine kurze Analyse der acht internationalen Millenniums-Entwicklungsziele (MDG) der Vereinten Nationen wie auch der zehn wichtigsten Punkte des Aktionsprogramms 2015 der Bundesregierung empfohlen. Die entscheidende Fragestellung dabei lautet: Kann in einem Entwicklungsland, dessen regierende politische Klasse und staatstragende Eliten keine »Ownership« für ein umfassendes Reformprogramm der staatlichen und gesellschaftlichen Strukturen aufbringen, vor allem bei Justiz, Polizei, Militär, Ordnungsbehörden, Verwaltung, parlamentarischer (oder vergleichbarer) Kontrolle, Partizipation der Zivilgesellschaft etc., überhaupt von einer nachhaltig wirksamen und erfolgversprechenden internationalen Entwicklungszusammenarbeit ausgegangen werden?

Von den zehn Punkten des Aktionsprogramms 2015 sind nur die Punkte 3. und 4. verifizierbar, da ihre Durchführung, wie z.B. »faire Handelschancen schaffen« oder »Entwicklung finanzieren « allein auf dem Willen und Mitteleinsatz der Geberländer beruht. Die übrigen Punkte wie z.b. »Gleichberechtigung der Geschlechter fördern«, »Menschenrechte verwirklichen « oder »Konflikte friedlich austragen « sind nach den Regeln praktischer Vernunft nicht umsetzbar, wenn nicht zuvor in dem Partnerland ein Rechtsstaat mit funktionierendem Sicherheitssektor eingerichtet wurde (oder realistische Ansätze hierzu unternommen wurden) und die Machteliten des Partnerstaates aus eigenem Antrieb und Vermögen an einer langfristig wirksamen und demokratischen Kontrolle des Sicherheitssektors festhalten.

Für die Entwicklungsziele der VN, genannt MDG, gilt dies ebenso. Von den acht Zielen erscheint prima facie keines in vollem Umfang erreichbar, ohne dass in einem betroffenen Land bzw. in einer Region zuvor die Voraussetzungen für eine transparente und kontrollierbare Umsetzung durch eine effiziente Verwaltung und dem Gesetz unterworfene Staatsstruktur geschaffen wurden. Kann man wirklich davon überzeugt sein oder behaupten wollen, dass kleine und reiche Oberschichten, die in korrupten Staatswesen abgekoppelt von der Mehrheit der Bevölkerung und geschützt durch unterbezahlte und abhängige Justiz und Polizei regieren, aus eigenem Antrieb tatkräftig und nachhaltig für die Überwindung der in den MDG genannten Misere eintreten würden? Hier ist glaubwürdige Hilfe von außen anzubieten. Eine neue Herausforderung für die Entwicklungszusammenarbeit wird erkennbar, die mit den bisherigen Instrumenten und Verfahren nicht zu bewältigen sein wird.

\subsection{Neue Ansätze in der deutschen Entwicklungs-Community}

Auch wenn in der deutschen Ministerialbürokratie die Weichen für ein konsequentes und unkonventionelles Herangehen an das Thema der »menschlichen Sicherheit « noch nicht gestellt werden konnten, ist doch Bewegung in 
die Vorfeldorganisationen und staatlich angelehnten Stiftungen gekommen. Im »Policy Paper 23 « der Stiftung Entwicklung und Frieden ist ein bemerkenswerter Spagat zwischen altem Denken und klischeehaftem Misstrauen gegenüber Militär und Polizei auf der einen und neuen Ansätzen in Richtung auf »Security First« gelungen. »Bisher gibt es kein Konzept der Bundesregierung zum Thema Umgang mit fragilen Staaten ... die Förderung von Good Governance, insbesondere die nachhaltige Stärkung hinreichend legitimer staatlicher Strukturen (state-building) dürfte künftig noch stärker als bisher eine Kernaufgabe der internationalen Organisationen und bilateralen Geber sein. « Und zur mangelnden Kooperation der Ressorts untereinander stellen die Autoren fest: "Dies lässt sich am Beispiel der Reform des Sicherheitssektors illustrieren, wo einzelne Ressorts der Bundesregierung de facto eine Reihe von Maßnahmen in anderen Staaten durchführen und finanzieren, ohne dass diese jedoch einer gemeinsamen Logik und Zielsetzung folgen." Ihrer Empfehlung kann man nur zustimmen: „Für Bundestag und Bundesregierung ist es dringend an der Zeit, eine Strategie zum Umgang mit »schwachen« und »versagenden« Staaten zu entwickeln. «12

Die Autoren regen auch eine Einteilung in Ländertypen an, um die unterschiedlichen Ausprägungen von Staatsversagen und Staatszerfall zu verstehen. Gerade solche Fälle, »in denen Staatlichkeit auf der Kippe steht, sollten im Zentrum der Außen-, Sicherheits- und Entwicklungspolitik stehen, um zu einer dauerhaften Stabilisierung im Sinne mittel- und langfristiger Krisenprävention beitragen zu können«. Endlich wird der nachvollziehbare Zusammenhang zwischen echter Krisenprävention auf der einen und nachhaltigen Governance-Aktivitäten in den bisher »unberührbaren Sektoren « des Staates auf der anderen hergestellt.

12 Tobias Debiel, Stephan Klingebiel, Andreas Mehler und Ulrich Schneckener, Zwischen Ignorieren und Intervenieren in fragilen Staaten, Stiftung Entwicklung und Frieden, Policy Paper 23, Bonn 2005, S. 10, http://www.sef-bonn.org/download/publikationen/policy_paper/pp_23_de.pdf.
Ein erster Schritt könnte darin liegen, in der Entwicklungszusammenarbeit zwischen sog. Post-Konflikt-Staaten und sog. »schwachen Staaten« zu unterscheiden. Bei den erstgenannten könnte man die Prinzipien von Security Sector Reconstruction anwenden, bei den letzteren könnten präventive Überlegungen im Vordergrund stehen, um Staaten mit Schwachstellen in ihrem Staatsaufbau wieder in stabile Verhältnisse zurückzubegleiten. Die aktuellen Ereignisse zeigen auf, dass auch die USA mit der Aufstellung eines ZivilKorps einen american way $\mathrm{zu}$ integrierbaren SSR-Prozessen gehen wollen. Man hat erkannt, dass langfristig wirksame Terrorismusbekämpfung in und mit Entwicklungsländern nur über deren stabilisierte Staatsstrukturen mit demokratisch kontrolliertem Sicherheitssektor möglich sein kann.

\section{Zusammenfassung}

Im Interesse der Steuerzahler in den Geberländern wie auch der in Unsicherheit lebenden Mehrheit der Weltbevölkerung ist es Zeit zum Umdenken: Es gilt, die Lebenslüge der Entwicklungszusammenarbeit zu überwinden, die da lautet, man könne die nachhaltige politische und ökonomische Umgestaltung eines Entwicklungslandes ohne die zuvor eingeleitete oder erreichte menschliche Sicherheit bewältigen. Dies kann nur erreicht werden mit einem demokratisch kontrollierten Sicherheitssektor, durch den ein jeglicher Staat sein legitimes Gewaltmonopol aufrechterhält. Die Entwicklungszusammenarbeit sollte dem Rechnung tragen und ihre beachtlichen Fähigkeiten in Governance-Beratungen zur Überwindung schwacher Staatsstrukturen konzentrieren. Damit würde man tatsächlich die marginalisierte Mehrheit der Weltbevölkerung in ihrem Streben nach mehr persönlicher Sicherheit unterstützen. Und erst danach rückte die Erreichung der Millennium Development Goals, auf die sich alle Staatschefs der UN-Mitgliedsländer verpflichtet haben, in den Bereich realer Machbarkeit.

Das gesamte Nomos Programm

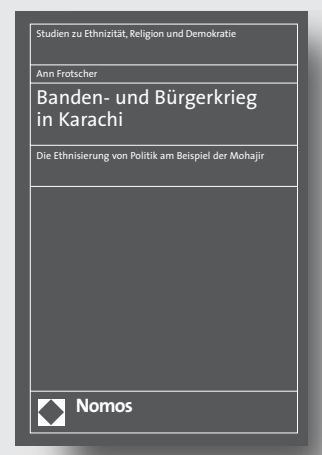

\section{Banden- und Bürgerkrieg in Karachi}

Die Ethnisierung von Politik am Beispiel der Mohajir

Von Dr. Ann Frotscher, Political Officer of the United Nations Field Missions

2005, 288 S., brosch., 59,- $€$, ISBN 3-8329-1100-6

(Studien zu Ethnizität, Religion und Demokratie, Bd. 6)

In dieser Arbeit wird die Gewalteskalation in Karachi seit Mitte der 8oer Jahre analysiert. Im Zentrum des Interesses stehen dabei die Identitätsbildung und Politisierung der Mohajir und ihrer Partei, der MOM. 